\title{
Genetic dissection of growth traits in a Chinese indigenous $\times$ commercial broiler chicken cross
}

\author{
Zheya Sheng ${ }^{1,2}$, Mats E Pettersson², Xiaoxiang $\mathrm{Hu}^{1}$, Chenglong $\mathrm{Luo}^{3}, \mathrm{Hao} \mathrm{Qu}^{3}$, Dingming Shu ${ }^{3}$, Xia Shen², \\ Örjan Carlborg ${ }^{2 *}$ and Ning Li $i^{* *}$
}

\begin{abstract}
Background: In China, consumers often prefer indigenous broiler chickens over commercial breeds, as they have characteristic meat qualities requested within traditional culinary customs. However, the growth-rate of these indigenous breeds is slower than that of the commercial broilers, which means they have not yet reached their full economic value. Therefore, combining the valuable meat quality of the native chickens with the efficiency of the commercial broilers is of interest. In this study, we generated an $F_{2}$ intercross between the slow growing native broiler breed, Huiyang Beard chicken, and the fast growing commercial broiler breed, High Quality chicken Line A, and used it to map loci explaining the difference in growth rate between these breeds.
\end{abstract}

Results: A genome scan to identify main-effect loci affecting 24 growth-related traits revealed nine distinct QTL on six chromosomes. Many QTL were pleiotropic and conformed to the correlation patterns observed between phenotypes. Most of the mapped QTL were found in locations where growth QTL have been reported in other populations, although the effects were greater in this population. A genome scan for pairs of interacting loci identified a number of additional QTL in 10 other genomic regions. The epistatic pairs explained 6-8\% of the residual phenotypic variance. Seven of the 10 epistatic QTL mapped in regions containing candidate genes in the ubiquitin mediated proteolysis pathway, suggesting the importance of this pathway in the regulation of growth in this chicken population.

Conclusions: The main-effect QTL detected using a standard one-dimensional genome scan accounted for a significant fraction of the observed phenotypic variance in this population. Furthermore, genes in known pathways present interesting candidates for further exploration. This study has thus located several QTL regions as promising candidates for further study, which will increase our understanding of the genetic mechanisms underlying growth-related traits in chickens.

Keywords: QTL, Indigenous chicken, Growth, Epistasis, SNP chip

\section{Background}

China has a wide variety of indigenous chicken breeds, most of which can only be found locally in rural areas. Because these breeds are geographically dispersed and have not been subjected to intense artificial breeding, they display unique characteristics as a result of the local environment and/or different breeding objectives than for commercial chicken. Because of the different traditional

\footnotetext{
*Correspondence: Orjan. Carlborg@slu.se; ninglcau@cau.edu.cn ${ }^{2}$ Division of Computational Genetics, Department of Clinical Sciences, Swedish University of Agricultural Sciences (SLU), Uppsala, Sweden ${ }^{1}$ State Key Laboratory for Agro-Biotechnology, China Agricultural University, Beijing, People's Republic of China

Full list of author information is available at the end of the article
}

food cultures across China, native broiler chickens have meat quality characteristics that are often favoured by consumers over those of commercial breeds. Several previous studies found that such characteristics include greater tenderness and preferred flavours [1-3]. Therefore, the native chicken breeds not only contribute to the conservation of poultry genetic resources, but are also of high economic value.

To evaluate the potential for genetic improvement of the productive efficiency of the native Huiyang Beard chicken (HB) breed while maintaining its valuable market properties, including high meat quality and unique appearance, we established an $F_{2}$ intercross between the slow-growing $\mathrm{HB}$ and the fast-growing commercial broiler breed "High 
Quality chicken Line A" (HQLA). We aimed to identify genes contributing to differences in productivity between these populations. Our first objective was to explore the genetic basis of the measured growth-related traits in this $\mathrm{F}_{2}$ cross.

In broilers, most economically important traits are growth-related. In this study we focused on six groups of such traits, which we expect to have been under positive selection during the commercial breeding of broiler chickens, including body weight at different ages, and efficiency of feed conversion. QTL mapping has previously been used to determine genomic regions affecting such quantitative traits in other mapping populations, and has identified a large number of QTL [4-7].

As these QTL are generally mapped with low marker density, lack of the genomic information could lead to the less accurately estimated recombination frequency or fail in detecting all the recombination events within the cross. Therefore, additional efforts by utilizing the recently developed genotyping platforms, which can rapidly and economically genotype a high density of SNP markers and have been widely applied to major farm animal species, such as cattle [8], pigs [9] and chickens [10], can be helpful in replicating and confirming these QTL.

In the last few years, the interest in identifying epistatic QTL effects has increased. Epistasis is when the combination of alleles at two, or more, loci yields a phenotype that cannot be explained by the independent effects of the involved loci [11-15]. A number of epistatic QTL have been identified in chicken for growth traits $[6,7,16,17]$, and here we further explored the importance of geneby-gene interactions in the genetic architecture of growth traits in this intercross.

The aim of the present study was thus to identify the main loci contributing to the phenotypic growth variability in this indigenous $\times$ commercial broiler $\mathrm{F}_{2}$ intercross, and estimate their direct and epistatic effects by conducting a genome-wide linkage analysis.

\section{Results}

All phenotypic measurements, units, and abbreviations are summarised in Table 1.

\section{Trait correlations}

The Pearson's correlations between all pairs of phenotypes conformed to expectations, but they varied in strength (Additional file 1: Table S1). There were high and positive correlations $(r>0.5)$ between live body weight $(B W)$, growth rate (GR), shank length (SL), and shank circumference (SC). Feed conversion ratio (FCR) was negatively correlated $(r>-0.5)$ with stomach weight (SW), BW and GR, and positively correlated with abdominal fat weight (AFW). AFW was negatively correlated $(r=-0.36)$ with $\mathrm{SL}$ at the later stages of growth. All the correlations
Table 1 Trait measurements and abbreviations used in this study

\begin{tabular}{ll}
\hline Trait, units & Abbreviation \\
\hline Live body weight at 2 weeks of age, g & BW2 \\
Live body weight at 4 weeks of age, g & BW4 \\
Live body weight at 6 weeks of age, g & BW6 \\
Live body weight at 8 weeks of age, g & BW8 \\
Live body weight at 10 weeks of age, g & BW10 \\
Live body weight at 12 weeks of age, g & BW12 \\
Growth rate at $0-4$ weeks of age, g & GR 0-4 \\
Growth rate at $4-8$ weeks of age, g & GR 4-8 \\
Growth rate at $8-12$ weeks of age, g & GR 8-12 \\
Shank circumference at 4 weeks of age, cm & SC4 \\
Shank circumference at 6 weeks of age, cm & SC6 \\
Shank circumference at 8 weeks of age, cm & SC8 \\
Shank circumference at 10 weeks of age, cm & SC10 \\
Shank circumference at 12 weeks of age, cm & SC12 \\
Shank length at 4 weeks of age, cm & SL4 \\
Shank length at 6 weeks of age, cm & SL6 \\
Shank length at 8 weeks of age, cm & SL8 \\
Shank length at 10 weeks of age, cm & SL10 \\
Shank length at 12 weeks of age, cm & SL12 \\
Stomach weight, g & SW \\
Abdominal fat weight, 9 & AFW \\
Feed conversion ratio at $6-8$ weeks of age & FCR 6-8 \\
Feed conversion ratio at $8-10$ weeks of age & FCR 8-10 \\
Feed conversion ratio at $10-12$ weeks of age & FCR 10-12 \\
\hline
\end{tabular}

mentioned above were significantly different from zero $(\mathrm{p}<0.001)$.

\section{One-dimensional QTL scan}

We performed QTL analyses for 24 traits related to bodysize in chickens. Forty-four QTL were detected for 22 traits (no QTL were found for GR 8-12 and FCR 10-12) on six chromosomes at two different significance levels. Five QTL were genome-wide significant at the $5 \%$ level, whereas all others were significant at the $1 \%$ level (Table 2; Additional file 1: Table S2). Figure 1a shows the genomewide QTL profile for BW10, while Figure $1 \mathrm{~b}$ and 1c present the QTL profiles for chromosomes 1 and 27 for several selected traits from all six groups (BW, GR, SC, SL, FCR and Carcass traits).

As shown in Table 2, most of the detected QTL clustered to distinct locations in the genome. The exception were the QTL mapped on GGA27, where peaks were distributed across two overlapping clusters - one cluster centred around $13 \mathrm{cM}$ and the other around $30 \mathrm{cM}$. Fitting both these locations in a two-QTL model did not 
Table 2 QTL affecting growth traits measured in this study

\begin{tabular}{|c|c|c|c|c|c|c|}
\hline QTL & Trait & QTL (cM) & Peak marker & $\mathrm{F}$ value $^{1}$ & Additive effect \pm SE & Var\% ${ }^{2}$ \\
\hline \multirow[t]{4}{*}{ CAU_AB 1a } & BW6 & 94 & rs13849470 & $11.7^{* *}$ & $28.8 \pm 5.9$ & $3.9 \%$ \\
\hline & BW8 & 89 & rs15225667 & $12.3^{* *}$ & $42.9 \pm 8.7$ & $3.9 \%$ \\
\hline & BW10 & 89 & rs15225667 & $11.4^{*}$ & $54.6 \pm 11.6$ & $3.3 \%$ \\
\hline & BW12 & 111 & rs13858917 & $13.0^{* *}$ & $76.0 \pm 15.1$ & $3.8 \%$ \\
\hline CAU_AB 1b & SL12 & 250 & rs13910430 & $11.6^{*}$ & $-0.84 \pm 0.21$ & $3.0 \%$ \\
\hline \multirow[t]{18}{*}{ CAU_AB 1C } & BW2 & 389 & rs13552715 & $14.6^{* *}$ & $6.3 \pm 1.2$ & $5.3 \%$ \\
\hline & BW4 & 399 & rs13974249 & $30.3^{* *}$ & $26.2 \pm 3.5$ & $10.8 \%$ \\
\hline & BW6 & 398 & rs15501880 & $36.9 * *$ & $53.9 \pm 6.4$ & $12.9 \%$ \\
\hline & BW8 & 398 & rs15501880 & $43.4^{* *}$ & $89.0 \pm 9.7$ & $14.9 \%$ \\
\hline & BW10 & 392 & rs13972116 & $47.4^{* *}$ & $125.6 \pm 13.0$ & $16.0 \%$ \\
\hline & BW12 & 392 & rs13972116 & $47.0^{* *}$ & $154.1 \pm 15.9$ & $15.9 \%$ \\
\hline & GR 0-4 & 399 & rs13974249 & $30.3^{* *}$ & $26.1 \pm 3.5$ & $10.8 \%$ \\
\hline & GR 4-8 & 392 & rs13972116 & $15.3^{* *}$ & $38.8 \pm 7.0$ & $5.6 \%$ \\
\hline & SC6 & 391 & rs14916980 & $26.7^{* *}$ & $0.06 \pm 0.01$ & $9.5 \%$ \\
\hline & $\mathrm{SC} 8$ & 391 & rs14916980 & $19.7^{* *}$ & $0.06 \pm 0.01$ & $7.1 \%$ \\
\hline & SC10 & 392 & rs13972116 & $30.8^{* *}$ & $0.07 \pm 0.01$ & $10.9 \%$ \\
\hline & $\mathrm{SC} 12$ & 392 & rs13972116 & $35.1^{* *}$ & $0.09 \pm 0.01$ & $12.3 \%$ \\
\hline & SL10 & 392 & rs13972116 & $20.9 * *$ & $1.4 \pm 0.21$ & $6.3 \%$ \\
\hline & SL12 & 392 & rs13972116 & $38.0^{* *}$ & $2.1 \pm 0.24$ & $10.9 \%$ \\
\hline & FCR 6-8 & 396 & rs13973293 & $18.8^{* *}$ & $-0.15 \pm 0.02$ & $6.8 \%$ \\
\hline & FCR 8-10 & 389 & rs13552715 & $17.2^{* *}$ & $-0.14 \pm 0.03$ & $6.2 \%$ \\
\hline & SW & 392 & rs13972116 & $44.5^{* *}$ & $2.2 \pm 0.24$ & $15.2 \%$ \\
\hline & AFW & 392 & rs13972116 & $47.0^{* *}$ & $-16.1 \pm 1.7$ & $16.0 \%$ \\
\hline CAU_AB 2a & SC4 & 213 & rs1422304 & $9.8^{*}$ & $-0.03 \pm 0.01$ & $3.5 \%$ \\
\hline CAU_AB 2b & BW2 & 281 & rs13794645 & $14.0^{* *}$ & $5.9 \pm 1.1$ & $4.8 \%$ \\
\hline CAU_AB 4 & SC6 & 152 & rs14499051 & $11.3^{*}$ & $-0.01 \pm 0.01$ & $3.4 \%$ \\
\hline CAU_AB 12 & AFW & 16 & rs14971272 & $12.3^{* *}$ & $-5.2 \pm 1.4$ & $3.5 \%$ \\
\hline \multirow[t]{15}{*}{ CAU_AB 27} & BW6 & 13 & rs13620303 & $18.9^{* *}$ & $31.6 \pm 6.2$ & $5.4 \%$ \\
\hline & BW8 & 13 & rs13620303 & $20.5^{* *}$ & $46.9 \pm 9.3$ & $5.8 \%$ \\
\hline & BW10 & 13 & rs13620303 & $17.6^{* *}$ & $56.0 \pm 12.3$ & $5.4 \%$ \\
\hline & BW12 & 13 & rs13620303 & $14.5^{* *}$ & $70.9 \pm 15.3$ & $4.4 \%$ \\
\hline & GR 4-8 & 11 & rs14302116 & $12.4^{* *}$ & $30.1 \pm 6.6$ & $4.3 \%$ \\
\hline & SC6 & 34 & rs15242584 & $19.2^{* *}$ & $0.05 \pm 0.01$ & $6.3 \%$ \\
\hline & SC8 & 30 & rs16047281 & $19.9^{* *}$ & $0.05 \pm 0.01$ & $6.7 \%$ \\
\hline & SC10 & 29 & rs16040742 & $23.9^{* *}$ & $0.06 \pm 0.01$ & $7.7 \%$ \\
\hline & $\mathrm{SC} 12$ & 30 & rs16047281 & $17.9^{* *}$ & $0.06 \pm 0.01$ & $5.7 \%$ \\
\hline & SL4 & 21 & rs15241178 & $13.1^{* *}$ & $0.63 \pm 0.13$ & $4.7 \%$ \\
\hline & SL6 & 32 & rs14303761 & $18.9^{* *}$ & $0.83 \pm 0.14$ & $6.9 \%$ \\
\hline & SL8 & 32 & rs14303761 & $23.1^{* *}$ & $1.1 \pm 0.17$ & $8.3 \%$ \\
\hline & SL10 & 32 & rs14303761 & $49.5^{* *}$ & $2.0 \pm 0.20$ & $16.6 \%$ \\
\hline & SL12 & 33 & rs16207882 & $53.0^{* *}$ & $2.4 \pm 0.23$ & $17.6 \%$ \\
\hline & AFW & 27 & rs16719300 & $17.3^{* *}$ & $-8.7 \pm 1.5$ & $5.3 \%$ \\
\hline \multirow[t]{2}{*}{ CAU_AB 28} & SC10 & 5 & rs16209969 & $12.0^{* *}$ & $-0.03 \pm 0.01$ & $3.5 \%$ \\
\hline & $\mathrm{SC} 12$ & 7 & rs15246230 & $11.9^{*}$ & $-0.04 \pm 0.01$ & $3.6 \%$ \\
\hline
\end{tabular}

\footnotetext{
$1{ }^{*} 5 \%$ genome-wide significance. ${ }^{* *} 1 \%$ genome-wide significance. ${ }^{2}$ the percentage of phenotypic variation explained by the detected QTL.
} 
allow us to discriminate whether there were two independent signals on the chromosome, or if they represented the same QTL with slightly shifted peaks for the different traits. Further analyses in other populations are needed to explore this region further.

The 44 detected QTL were categorized into nine independent QTL. We named each QTL by combining an abbreviation for the cross (China Agricultural University hqlA $\times$ hB; $\left.C A U_{-} A B\right)$ and the number of the chromosome where the QTL was located. In addition, when multiple distinct QTL were located on the same chromosome, letters were added at the end indicating their order along the chromosome. For example, $C A U_{-} A B$ 1a represent the most proximal QTL on GGA1.

Four out of the nine independent QTL affected several of the analyzed traits and were therefore classified as being pleiotropic. QTL $C A U \_A B 1 a$ affected the intermediate to late growth stage of $\mathrm{BW}$, while $C A U \_A B 28$ was associated with the late growth phase of SC. Two QTL
(CAU_AB 1c and $C A U \_A B$ 27) affected almost all measured growth traits in this cross, which was consistent with the fact that high correlations existed among the traits affected by these QTL. CAU_AB 1c, on the other hand, affected FCR and the early stage of BW, traits that were not significantly correlated. The remaining five QTL only affected single traits. For the traits recorded at multiple time-points in life, such as BW, SC, and SL, differences were found in the sets of QTL controlling the traits in the different growth stages. More QTL, with larger average effects, were found for growth at intermediate and late growth phases than for early growth.

Positive additive effects, implying enhanced growth associated with the alleles originating from the fast-growing HQLA line, were observed for most of the traits. Most of the exceptions (i.e. QTL with negative additive effects) were detected for AFW and SC. The HQLA line is leaner than $\mathrm{HB}$, and consequently this observation is consistent with the expectations, as a negative additive effect

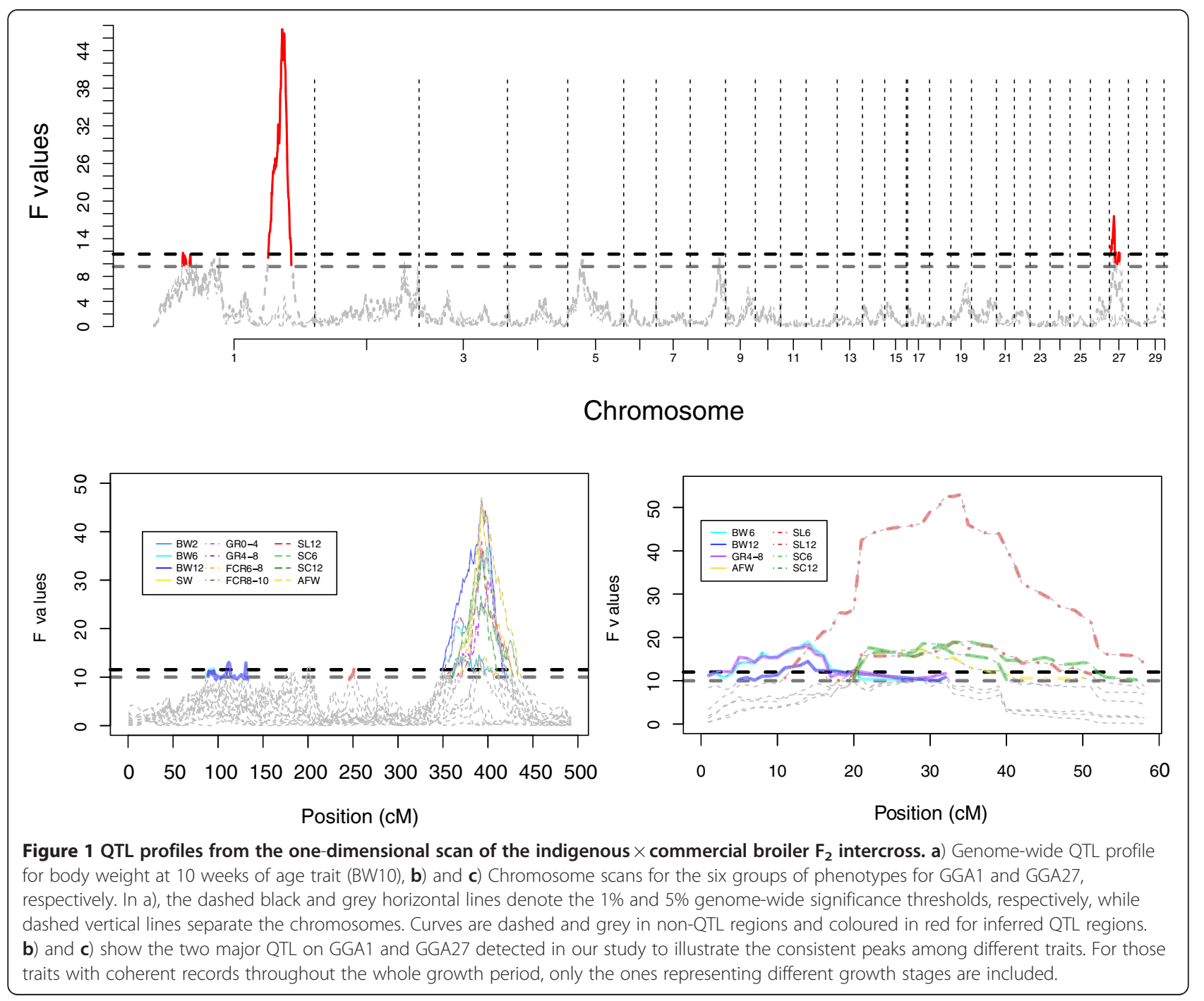


indicates that $\mathrm{HB}$ alleles increase AFW. Interestingly, both lines had positive effects on SC in different QTL regions. The proportions of residual phenotypic variance explained by the detected QTL ranged from 3-18\%, with the largest effects from $C A U_{-} A B 1 c$ and $C A U_{-} A B$ 27. It is worth to note that estimation of the genetic variance associated with the detected QTL is generally biased upward $[18,19]$. In a population of the size used here, only a slight upward bias is expected and therefore the estimates are provided to facilitate a comparison of the relative contribution of the inferred QTL to the observed phenotypic variance in this intercross population.

\section{Two-dimensional QTL scan}

\section{Epistatic QTL mapping}

We observed significant epistatic interactions for four traits in 10 distinct regions on 10 chromosomes (Table 3; Additional file 1: Table S3). By also examining suggestive interactions (Additional file 1: Table S4), we found that these belonged to coherent peaks, although they did not reach the significance threshold. Therefore, we defined the significant epistatic QTL pairs to be interactions between QTL-peak regions rather than interactions between single marker positions.

As shown in Additional file 1: Table S3, additiveby-additive interactions contributed a large proportion of the total epistatic effects for four out of the five interacting pairs. The only exception was for the pair affecting BW6, where the dominance-by-dominance effect was most significant.

(Additional file 2: Figure S2) shows the genotypephenotype map for the interacting pairs. Each pair had a unique epistatic pattern, indicating complicated interactions between the loci.

In summary, interactions were often identified in the same regions for consecutive growth phases of the BW traits, even though not all reached the significance threshold (Additional file 1: Table S4). This indicates that the identified interacting effects act throughout extended

Table 3 Significant QTL pairs in the two-dimensional epistatic scan in the indigenous $\times$ commercial $F 2$ population

\begin{tabular}{|c|c|c|c|c|c|c|}
\hline \multirow[t]{2}{*}{ Trait } & \multicolumn{2}{|c|}{ QTL1 $^{1}$} & \multicolumn{2}{|c|}{ QTL2 $^{2}$} & \multirow{2}{*}{$\begin{array}{l}\text { F } \\
\text { value }^{3}\end{array}$} & \multirow{2}{*}{$\begin{array}{l}\text { Var } \\
\%^{4}\end{array}$} \\
\hline & $\mathrm{Chr}^{1}$ & $\operatorname{Pos}^{1}(\mathrm{cM})$ & $\mathrm{Chr}^{2}$ & $\operatorname{Pos}^{2}(\mathrm{cM})$ & & \\
\hline BW6 & 4 & 55 & 7 & 100 & $9.8^{*}$ & $6.8 \%$ \\
\hline \multirow[t]{2}{*}{ GR 4-8 } & 6 & 40 & 25 & 60 & $9.5^{*}$ & $6.5 \%$ \\
\hline & 1 & 300 & 5 & 120 & $8.8^{+}$ & $6.1 \%$ \\
\hline FCR 6-8 & 3 & 215 & 26 & 40 & $11.1^{*}$ & $8.1 \%$ \\
\hline FCR 8-10 & 20 & 10 & 22 & 40 & $9.4^{*}$ & $6.4 \%$ \\
\hline
\end{tabular}

${ }^{1,2}$ The first ${ }^{1}$ and second ${ }^{2}$ QTL in the significant epistatic QTL pair and their chromosomal location in centimorgans (CM); ${ }^{3}{ }^{*} 5 \%$ genome-wide significance; $+10 \%$ suggestive genome-wide significance. ${ }^{4}$ The percentage of the residual phenotypic variance explained by the epistatic QTL pair. growth periods. The inferred epistatic pairs explained 6-8\% of the residual phenotypic variance, which is substantial given the size of the QTL detected in the single-QTL scan.

\section{Identifying candidate epistatic genes in known biological pathways}

In contrast to the results from the single-QTL scan, where correlated traits often shared the same QTL, the epistatic analysis identified mostly novel regions (Table 3 ). When examining the 10 epistatic regions in more detail [20], genes from the same KEGG biological pathway [21], ubiquitin mediated proteolysis (UMP), were found in seven of the 10 regions (Table 4). The genes belonging to the UMP pathway across the genome and in the identified epistatic regions are presented in Figure 2. Using a 10,000-fold permutation test, we estimated that the probability of observing such a seven-locus overlap with the gene from UMP pathway was only 1.6\% (Additional file 3: Figure S3). The UMP pathway is a temporally controlled and tightly regulated process, which plays major roles in a variety of basic pathways during cell life and death. Hence, it is crucial for cell growth and differentiation [22]. For the QTL pairs affecting FCR 6-8 and FCR 8-10, we also found possible interacting candidate genes in a growth-related pathway, the ErbB signalling pathway, which is known to affect intracellular signalling pathways regulating cell proliferation, differentiation, cell motility, and survival [23]. Genes in the ErbB pathway were also identified in the epistatic regions for GR 4-8.

\section{Discussion}

This study describes the genetic dissection of 22 growth related traits in a chicken $F_{2}$ population. Forty-four QTL were identified, 39 of which belonged to one of four major pleiotropic QTL regions, while the remaining five each affected a single trait. The co-localized QTL did, however, explain different amounts of residual phenotypic variance for different traits. Given the correlations among traits, this high degree of pleiotropy observed for the QTL was not surprising. For instance, the HQLA allele at $C A U \_A B$ $1 c$ increases both growth rate and size of the digestive organs, a correlated response that has been observed in previous physiological studies $[24,25]$. The pleiotropic effects of this QTL on many traits investigated in this study are in the direction preferred in selection programs for increased productivity, as the HQLA allele improves the performance of traits like BW and SL, while at the same time decreasing AFW and FCR. These characteristics of the QTL make it an interesting candidate for further studies to better understand the molecular mechanisms underlying the response to selection for faster growth in chickens.

The QTL CAU_AB 1c, which affects many traits in this cross, is located at the distal end of GGA1 (173.7 Mb). 
Table 4 Candidate genes in the epistatic QTL regions and their involvement in growth-related pathways

\begin{tabular}{llllll}
\hline Trait & Gene & Chr & Position bp/(cM) & Pathway $^{\mathbf{2}}$ \\
\hline BW6 & UBE2A ubiquitin-conjugating enzyme E2A & 4 & $16506437-16512992(55-55.1)$ & UMP \\
& MARCH7 membrane-associated ring finger (C3HC4) 7 & 7 & $36187561-36207776(99.8)$ & UMP \\
GR 4-8 & HERC4 hect domain and RLD 4 & 6 & $10024961-10054292(36.7)$ & UMP \\
& ZBTB1 zinc finger and BTB domain containing 1 & 5 & $52791938-52805593(135.1)$ & UMP \\
& MID1 midline 1 & 1 & $124042084-124134384(296.9)$ & UMP \\
FCR 6-8 & RNF144A ring finger protein 144A & 3 & $94598486-94657682(206.8)$ & UMP \\
& NRAS neuroblastoma RAS viral (v-ras) oncogene homolog & 26 & $3847830-3854232(43.6)$ & ER \\
FCR 8-10 & ITCH itchy E3 ubiquitin protein ligase homolog & 20 & $1769346-1829955(7.5-7.7)$ & UMP & ER \\
\hline
\end{tabular}

${ }^{1}$ The physical locations of the genes based on the WASHUC 3.1 build, and the genetic locations of the scored SNP markers located within the gene. ${ }^{2}$ UMP: Ubiquitin-mediated proteolysis; ER: ErbB signalling.

This location overlaps with several earlier reported growth QTL in the Chicken QTL Database [26]. Interestingly, it also maps very near to a $1.5-\mathrm{Mb}$ region $(173.5-175 \mathrm{Mb}$ ) on GGA1, identified in a recent GWAS study examining growth differences between the White Recessive Rock and the Xinghua chicken (another Chinese indigenous breed) [27]. Most of the other QTL mapped in this study also overlap with earlier reported QTL [26]. We did, however, find that these loci have larger genetic effects and higher statistical significance in this study. This could be due to the more informative genetic markers used, but also a reflection of the larger divergence between the founder lines for the studied traits. This would, in turn, indicate that these loci have been important for the selection response while breeding broiler chickens and that there exist multiple, distinct alleles at these loci in the global chicken population. Further in-depth molecular dissection of these loci could therefore help to identify the genetic mechanisms underlying important traits in broiler breeding.

No significant result was found for GR 8-12, despite the strong signals detected for both BW8 and BW12. Also, no significant QTL was detected for FCR 10-12, which was consistent with the observation that GR 8-12 was strongly correlated with FCR 10-12. This result indicates that late GR is not controlled by the same loci as early GR, and that fewer loci with large individual effects
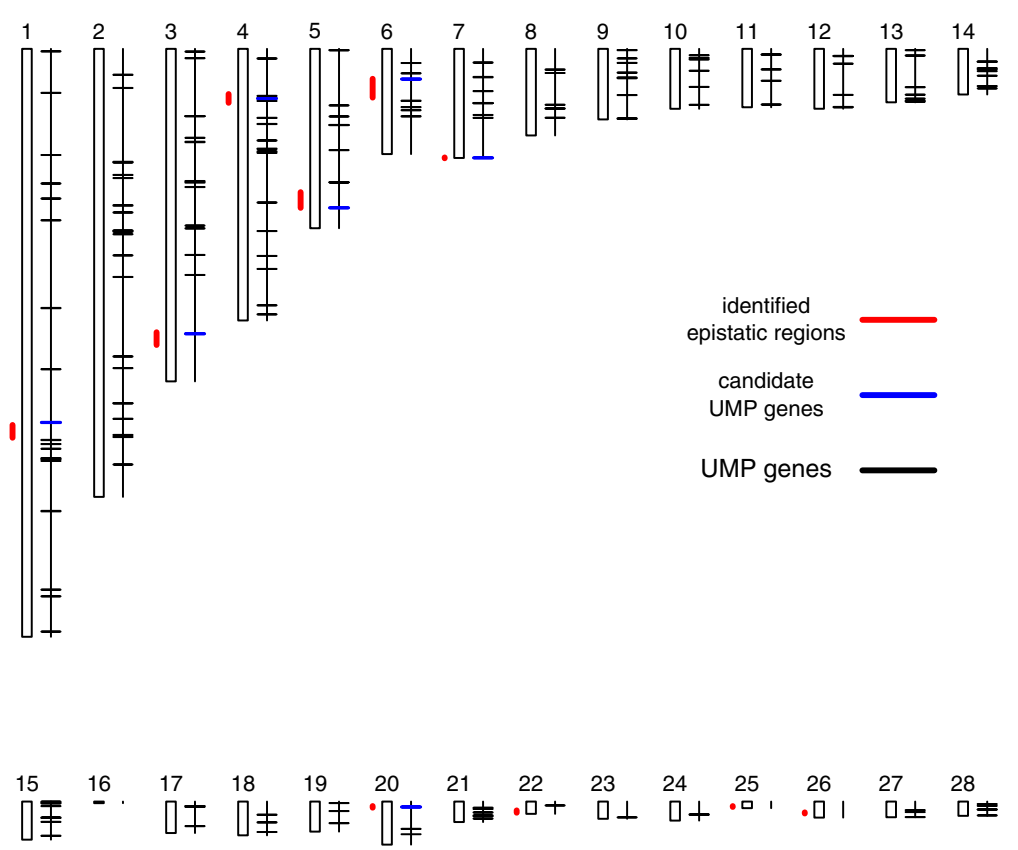

Figure 2 Localisation of epistatic QTL and corresponding candidate genes from the UMP pathway throughout the genome. The 28 vertical boxes represent chicken autosomes GGA1-GGA28. All 10 identified epistatic regions are presented as red vertical bars, and UMP genes within the epistatic regions are presented as blue horizontal lines, while other genes are displayed in black. 
appear to be involved in late GR, which is consistent with earlier findings in chicken [16].

The epistatic analysis identified significant interactions for four traits, all affecting the birds between 6-10 weeks of age. This is the same time-period where the highest statistical significance for QTL was found also in the single locus analysis. The epistatic QTL identified at different times appeared to have a much more complicated pattern than that revealed in the single-QTL scan. Even so, for some traits such as BW, we could conclude that the same epistatic QTL pairs were identified for late growth (from 6-10 weeks of age; Additional file 1: Table S4), while different QTL pairs were found for early growth (from 2-4 weeks of age, data not shown). Both the results from the epistatic analysis and the single locus scan are consistent with earlier findings that different sets of genes regulate early and late growth [16,28-30].

(Additional file 2: Figure S2) shows the two-locus genotype-phenotype maps for the detected epistatic pairs. All displayed complicated interaction patterns, two of which were found to be more intriguing. These two pairs affected BW6 and GR84 (Additional file 2: Figure S2a and S2c) and had significantly larger phenotype values when both the interacting QTL were homozygous for the allele with a HQLA origin. This pattern is consistent with the selective breeding scheme that has been applied in the founder lines. Also, it suggests that selection for growth is not acting only on independent alleles at single loci, but also on combinations of alleles at multiple loci.

Compared to the single-QTL analysis, the relatively large genetic variances explained by the pairs detected in the epistatic analysis suggested that epistasis is an important contributor as some of the pairs explained more than the minor single QTL effects. Interestingly, the five epistatic QTL pairs involved 10 independent regions across 10 autosomes, indicating that the detected epistatic loci each provide novel insights into the genetic basis of the studied growth traits. It is somewhat surprising that none of the highly significant QTL detected in one-dimensional analysis were involved in significant epistatic interaction with other regions across the genome. This low degree of overlap between QTL identified in one- and two-dimensional scans, however, illustrates the need to perform both single-QTL and epistatic QTL analysis to better dissect complex quantitative traits.

Most of the epistatic regions contained genes from the UMP, or related, pathways. The UMP pathway contains 204 genes across the chicken genome (Additional file 1: Table S5) $[20,21]$. A permutation test estimated that the probability of obtaining such an overlap by chance was about 1.6\% (Additional file 3: Figure S3), indicating that this result is unlikely to be a coincidence. Further studies of this pathway will hopefully be able to provide new insights to the genetics of growth-related phenotypes.
Because of its cost efficiency and ease of use, we genotyped the birds using a dense SNP chip. While the high marker density does not immediately help to narrow QTL regions because of the lack of recombination events in the F2 design, it does help to increase the precision of the line origin estimation. Much work remains to identify the causal mutations underlying the traits, but two regions are of particular interest. Further studies of the $C A U_{-} A B \quad 1 c$ region on GGA1 and the entire GGA27 are highly promising, as the effects at those loci are particularly strong. In addition, the epistatic analysis identified interesting QTL regions where a set of possible candidate genes that are part of the same biological pathway are located. As sequencing technology becomes more affordable, re-sequencing can be used to search for potential functional polymorphisms between the lines in the highlighted regions as a way to identify the causal genes.

\section{Conclusion}

In summary, we performed a genome-wide QTL analysis in an indigenous $\times$ commercial broiler $\mathrm{F}_{2}$ intercross. A singleQTL analysis revealed nine distinct QTL regions with significant effects on 22 traits. These QTL were often pleiotropic, which is consistent with the observed correlations between the studied traits, and mostly mapped to regions identified in earlier chicken studies. The genetic effects of these loci were, however, greater in this study than in previous reports. Second, an exhaustive search for epistatic effects identified five distinct interacting QTL pairs. The epistatic QTL did not, however, overlap with the results of the single-QTL analysis, which indicates that epistatic combinations of loci might have contributed to selection response in addition to the single QTL, but that these are more dependent on the allelic combinations available in the founders. For seven of the 10 epistatic QTL, we found candidate genes belonging to the UMP pathway, suggesting this as a potentially important pathway in the regulation of growth in chicken.

\section{Methods}

\section{Ethics statement}

All animals used in the current study were cared for and used according to the requirements of the Institute of Animal Science, Guangdong Academy of Agricultural Sciences (No. GAAS-IAS-2009-73).

\section{Experimental population}

A three-generation intercross population was constructed from two divergent chicken lines. One of the founder lines was the "High Quality chicken Line A" (HQLA), bred by Guangdong Wiz Agricultural Science \& Technology Co. (Guangzhou, China), a closed population founded by the commercial Anak chicken breed and an indigenous chicken line unrelated to the other founder line of this 
study. This population has been under selection for fast growth for more than 10 generations, while maintaining the meat quality. The other founder line was the Huiyang Beard chicken (HB), a native Chinese meat-type breed, which is characterized by slow growth and high meat quality. The average phenotypes, i.e. body weight at consecutive growth phases, are given for each sex in each line in (Additional file 1: Table S6).

The $F_{2}$ cross was generated by reciprocal crossing of the founder lines (details presented in Additional file 4: Figure S4). We denote the cross between a cock from line HQLA and a hen from line $\mathrm{HB}$ as cross type $\mathrm{A} \times \mathrm{B}$, the reverse as cross type $\mathrm{B} \times \mathrm{A}$. First, four cocks and 12 hens from each line were chosen as the $F_{0}$ generation. The males were full siblings, while the females were either half or full siblings. It is important to note that the cocks and hens of the same line did not share either of their direct parents, thus not closely related. Each cock was mated to three hens and $399 F_{1}$ individuals were produced. To balance the offspring of the $8 \mathrm{~F}_{0}$ cocks in the next generation, 56 chickens from the $F_{1}$ population, all with good health condition and similar body weight, were selected for further crossing. Each cock from cross type $\mathrm{A} \times \mathrm{B}$ was mated with six hens from cross type $\mathrm{B} \times \mathrm{A}$ and vice versa. Forty-eight full-sib families including $800 \mathrm{~F}_{2}$ individuals were produced and hatched in six batches.

During the first 5 weeks, the chickens were divided into groups by hatch. Each group was kept in a single cage. A starter diet $(2,900 \mathrm{kcal}$ of $\mathrm{ME} / \mathrm{kg}$ and $200 \mathrm{~g} / \mathrm{kg}$ of CP) was provided during this period. Then, from weeks $6-13$, each individual was reared separately and fed a grower diet (2,950 kcal of ME/ $\mathrm{kg}$ and $180 \mathrm{~g} / \mathrm{kg}$ of CP). All chickens had free access to feed and water. The breeding facility supplied 24-hour lighting and was equipped with a water curtain system to control the ambient temperature.

\section{Phenotyping}

BW was measured at hatching and every other week until 12 weeks of age. Using these observations, we calculated three estimates of GR as the weight gain during the periods, which were $0-4,4-8$, and $8-12$ weeks of age. During weeks 4-12, SL and SC were also measured every 2 weeks. Anatomical traits such as SW (the combined weight of the ventriculus and the proventriculus) and AFW were recorded at the 13th week, after the birds were slaughtered. FCR was calculated as the ratio between feed intake and body weight gain over the weeks specified.

Boxplots for each phenotype were generated in $\mathrm{R}$ [31] to scan for outliers. Individuals that were further than 1.5 times the interquartile range away from the lower or upper quartile of the boxplots were marked for further examination. The majority of the marked data were considered as outliers, and were eliminated from further analysis (data not shown). However, the marked data were not excluded if there were multiple marked data points that were clustered, or if the data points were observed consistently throughout the growth phase. After this procedure, descriptive statistics of the phenotypes in the population were calculated and are provided in Table 5.

\section{Genotyping and map construction}

In total, 585 individuals were genotyped, comprising 22 $\mathrm{F}_{0}$ individuals, $52 \mathrm{~F}_{1}$ animals, and $511 \mathrm{~F}_{2}$ progeny (from 43 of the 48 full-sib families). Families were selected for genotyping were those with the highest quality of the pedigree information and phenotype records. Genomic DNA extraction from blood was performed using the phenol-chloroform method. Genotyping was performed using an Illumina Chicken 60K SNP Beadchip [10] and was performed by DNA LandMarks (Saint-Jean-sur-Richelieu, Canada).

Quality control was assessed using custom Perl [http:// www.perl.org/] scripts (available from the authors on

Table 5 Descriptive statistics for the studied phenotypes

\begin{tabular}{|c|c|c|c|c|c|}
\hline Traits & No. of records & Mean & SD & Minimum & Maximum \\
\hline BW2 (g) & 493 & 167.2 & 21.6 & 115.0 & 251.0 \\
\hline BW4 (g) & 491 & 443.5 & 74.2 & 273.0 & 708.5 \\
\hline BW6 $(g)$ & 492 & 803.1 & 131.6 & 439.0 & 1290.5 \\
\hline BW8 $(g)$ & 492 & 1240.1 & 208.5 & 475.5 & 2005.5 \\
\hline$B W 10(g)$ & 493 & 1662.0 & 287.2 & 727.5 & 2719.5 \\
\hline BW12 (g) & 492 & 2032.2 & 352.4 & 1042.0 & 3250.0 \\
\hline GR 0-4 (g) & 490 & 415.9 & 73.5 & 246.4 & 676.8 \\
\hline GR 4-8 (g) & 490 & 798.3 & 154.5 & 110.5 & 1297.0 \\
\hline GR 8-12 (g) & 491 & 792.5 & 177.7 & 20.5 & 1277.5 \\
\hline $\mathrm{SC4}(\mathrm{cm})$ & 493 & 2.9 & 0.25 & 2.2 & 3.5 \\
\hline $\mathrm{sc6}(\mathrm{cm})$ & 493 & 3.4 & 0.27 & 2.7 & 4.1 \\
\hline $\mathrm{SC}(\mathrm{cm})$ & 493 & 3.8 & 0.31 & 2.9 & 4.5 \\
\hline $\mathrm{SC10}(\mathrm{cm})$ & 493 & 4.0 & 0.32 & 3.3 & 4.7 \\
\hline $\mathrm{SC12}(\mathrm{cm})$ & 493 & 4.2 & 0.36 & 3.1 & 5.0 \\
\hline SL4 $(\mathrm{mm})$ & 493 & 54.9 & 3.9 & 43.0 & 63.5 \\
\hline SL6 $(\mathrm{mm})$ & 493 & 68.2 & 4.7 & 55.5 & 80.7 \\
\hline SL8 $(\mathrm{mm})$ & 493 & 80.4 & 6.2 & 61.6 & 97.2 \\
\hline SL10 $(\mathrm{mm})$ & 493 & 89.3 & 8.2 & 68.1 & 106.4 \\
\hline SL12 $(\mathrm{mm})$ & 493 & 92.8 & 10.2 & 73.5 & 114.3 \\
\hline$S W(g)$ & 493 & 19.1 & 3.9 & 10.2 & 36.3 \\
\hline$A F W(g)$ & 490 & 83.3 & 33.7 & 2.3 & 169.1 \\
\hline FCR 6-8 & 490 & 2.9 & 0.39 & 1.9 & 7.1 \\
\hline FCR 8-10 & 492 & 3.5 & 0.46 & 2.1 & 5.6 \\
\hline FCR $10-12$ & 486 & 4.4 & 0.76 & 2.9 & 11.1 \\
\hline
\end{tabular}


Table 6 Summary statistics for the linkage map and number of informative markers in the $\mathrm{F} 2$ population

\begin{tabular}{|c|c|c|c|c|}
\hline Chromosome & $\begin{array}{l}\text { Physical } \\
\text { size1 (Mb) }^{1}\end{array}$ & $\begin{array}{l}\text { Number } \\
\text { of SNPs }\end{array}$ & $\begin{array}{l}\text { Sex } \\
\text { average (cM) }\end{array}$ & $\begin{array}{l}\text { Recombination } \\
\text { rate }(\mathrm{cM} / \mathrm{Mb})^{2}\end{array}$ \\
\hline GGA1 & 201 & 6800 & 491.2 & 2.4 \\
\hline GGA2 & 155 & 5073 & 318.8 & 2.1 \\
\hline GGA3 & 114 & 3924 & 269.3 & 2.4 \\
\hline GGA4 & 94 & 3143 & 183 & 2 \\
\hline GGA5 & 62 & 2112 & 170.1 & 2.7 \\
\hline GGA6 & 37 & 1595 & 98.8 & 2.7 \\
\hline GGA7 & 38 & 1627 & 103.9 & 2.7 \\
\hline GGA8 & 31 & 1330 & 107.4 & 3.5 \\
\hline GGA9 & 26 & 1115 & 90.2 & 3.5 \\
\hline GGA10 & 22.6 & 1247 & 77 & 3.4 \\
\hline GGA11 & 21.9 & 1172 & 77.2 & 3.5 \\
\hline GGA12 & 20.5 & 1252 & 94.1 & 4.6 \\
\hline GGA13 & 18.9 & 1090 & 76.8 & 4.1 \\
\hline GGA14 & 15.8 & 968 & 67.1 & 4.3 \\
\hline GGA15 & 13 & 993 & 65.9 & 5.1 \\
\hline GGA16 & 0.43 & 13 & 0.8 & n.d. ${ }^{3}$ \\
\hline GGA17 & 11.2 & 830 & 66.8 & 6 \\
\hline GGA18 & 10.9 & 826 & 65 & 6 \\
\hline GGA19 & 9.9 & 779 & 62.8 & 6.3 \\
\hline GGA20 & 14 & 1346 & 75.2 & 5.4 \\
\hline GGA21 & 7 & 730 & 54.2 & 7.7 \\
\hline GGA22 & 3.9 & 285 & 47.4 & 12.2 \\
\hline GGA23 & 6 & 562 & 60.2 & 10 \\
\hline GGA24 & 6.4 & 659 & 60.6 & 9.5 \\
\hline GGA25 & 2.03 & 164 & 61.8 & n.d. ${ }^{3}$ \\
\hline GGA26 & 5.1 & 601 & 57.5 & 11.3 \\
\hline GGA27 & 4.8 & 436 & 57.8 & 12.1 \\
\hline GGA28 & 4.5 & 503 & 55.5 & 12.3 \\
\hline LGE22 & 0.9 & 103 & 52 & n.d. ${ }^{2}$ \\
\hline $\begin{array}{l}\text { Total } \\
\text { autosomal }\end{array}$ & 957.8 & 41278 & 3068.4 & 3.2 \\
\hline
\end{tabular}

${ }^{1}$ Physical length of the chromosome was based on the WASHUC 3.1 build.

${ }^{2}$ Estimated from the genetic map estimated in this population and the physical map from the NCBI database. ${ }^{3}$ n.d. $=$ not determined, as the chromosome showed clear evidence of sequence gaps.

request). The QC procedure excluded 24 individuals and 16352 SNPs from the analysis for failing to fulfil one or more of the following criteria: call rate of individuals $>0.9$, call frequency of SNPs $>0.9$, minor allele frequency $>0.05$, and inheritance error rate for either individuals or SNPs $<0.05$ (The complete genotype data are available in Additional file 5).

An improved version of CRI-MAP [32] was used to construct and validate the genetic map. The result was a sex-average linkage map for 29 autosomal linkage groups spanning about $3068 \mathrm{cM}$, which is consistent with reported linkage maps in chickens [33]. Further details regarding the linkage map are presented in Table 6.

\section{Statistical analysis}

Preliminary models (as shown below) were fit to determine which non-genetic effects should be included in the further analysis of each trait.

$$
y=\mu+\beta F+\gamma C+\varepsilon
$$

Here, $y$ is the phenotypic value, $\mu$ is the mean of estimated phenotypic values, $\beta$ is the estimate for fixed effects, $\gamma$ is the estimated effects of covariates, $F$ and $C$ are the indicator variables of fixed effects and covariates, respectively, and $\varepsilon$ is a normally distributed residual error.

Although fixed effects, including sex, batch, and family effects, as well as the possible covariates, were considered, only those that had significant effects were included in further analyses (details are available in Additional file 1: Table S2).

\section{One-dimensional QTL scan}

Single QTL were mapped using the least squares regression method as described by Haley et al. (1994) [34]. Based on the genetic map of our population, the probabilities for the line origin combinations, that is, the probabilities of both alleles being inherited from line HQLA (genotype AA), both being inherited from line $\mathrm{HB}$ (genotype $\mathrm{BB}$ ), and one allele being inherited from each line (genotype $A B$ and $B A$ ), were calculated using the triM [35] algorithm at every centiMorgan $(\mathrm{cM})$ throughout the genome for all $\mathrm{F}_{2}$ individuals. Once the line origin probabilities had been calculated, the coefficients for $a$ and $d$ for a putative QTL at each cM can be determined as $A=\operatorname{Pr}($ genotype $A A)-\operatorname{Pr}$ (genotype $B B)$ and $D=\operatorname{Pr}($ genotype $A B)+\operatorname{Pr}($ genotype $B A)$, where $\operatorname{Pr}($ genotype $X)$ is the probability of having genotype $\mathrm{X}$. Then, a multiple linear regression model was used to estimate the genetic effects for putative QTL at $1 \mathrm{cM}$ intervals across the genome.

$$
y=\mu+\beta F+\gamma C+a A+d D+\varepsilon
$$

where $y, \mu, \beta, \gamma, F$, and $C$ are the same as stated in model (1), $a$ and $d$ are the additive and dominance effects, respectively, of the tested position, and $\varepsilon$ is a normally distributed residual error. $\mathrm{F}$ values were calculated based on model (1) and model (2).

Additive and dominance regression indicator variables of the most significant QTL in each round of analysis were added to the statistical model (2), and another genome scan was performed until no more significant QTL were detected for the analysed trait. 
To potentially separate effects of QTL on the same chromosome, we performed a second statistical analysis including both QTLs on the same chromosome in the model. If both QTL effects remained significant after this procedure, we defined them as independent QTL.

\section{Two-dimensional scan}

To explore the interacting effects ( $i$, as shown below in model (4)) between two loci, we further used the line origin probabilities to calculate the indicators of $i$ by multiplying the indicator variables from locus 1 with those from locus 2 (indicated in the subscripts). For instance, the coefficent for the additive-by-additive effect between two loci can be generated by the formula: $I_{A 1 A 2}=$ $A_{1} \times A_{2}=[\operatorname{Pr}($ genotype $A A$ for locus 1$)-\operatorname{Pr}($ genotype $B B$ for locus 1) $] \times[\operatorname{Pr}($ genotypeAA for locus 2$)-\operatorname{Pr}($ genotypeBB for locus 2)], where $\operatorname{Pr}($ genotypeX for locus $K$ ) is the probability of locus $\mathrm{K}$ having genotype $\mathrm{X}$.

On the basis of the two-locus models, including the null model (model (3)) and the full model (model (4)), an exhaustive two-dimensional genome scan was performed to detect pairs of epistatic QTL:

$$
\begin{aligned}
y= & \mu+\beta F+\gamma C+a_{1} A_{1}+d_{1} D_{1}+a_{2} A_{2} \\
& +d_{2} D_{2}+\varepsilon \\
y= & \mu+\beta F+\gamma C+a_{1} A_{1}+d_{1} D_{1}+a_{2} A_{2} \\
& +d_{2} D_{2}+i_{1} I_{A 1 A 2}+i_{2} I_{A 1 D 2}+i_{3} I_{D 1 A 2} \\
& +i_{4} I_{D 1 D 2}+\varepsilon
\end{aligned}
$$

where $y, \mu, \beta, \gamma, F$, and $C$ are as stated in model (1), $A_{1}, D_{1}$, $A_{2}$ and $D_{2}$ are the additive and dominance indicator variables of the first and second tested loci, $I$ values are the indicators of interacting effects, $a_{1}, d_{1}, a_{2}$ and $d_{2}$ are the additive and dominance effect, respectively, of the first and second locus (indicated in the subscripts), and $i_{1}, i_{2}, i_{3}$ and $i_{4}$ are the additive-by-additive, additive-by-dominance, dominance-by-additive, and dominance-by-dominance interaction effects between two positions. F values were calculated based on models (3) and (4).

The genotype-phenotype maps for detected epistatic pairs were visualised using a discretised estimate of the line origin. Here, only individuals for which the probability of one of the genotypes was higher than 0.8 were included. Then if, for example, $\operatorname{Pr}($ genotypeAA) for an individual at a marker was greater than 0.8 , it would be assigned the discretised genotype $A A$ at this marker. This set of individuals with high-confidence, discretised genotypes in the interacting regions was then used to estimate the residual (ỹ from model (1)) phenotypic means for each two-locus genotype.

The permutation test for the distribution of the number of regions containing genes from the UMP pathway was performed in three steps. First, we allocated each region to a chromosome by sampling with replacement in the range of 1-28, since the epistatic QTL scan was performed only on the 28 autosomes. Second, to best mimic our results, we selected the size of each region by sampling without replacement from the vector of detected regions sizes, found in Additional file 1: Table S3. Each size selection was followed by a validation step, to ensure that the chromosome in question was larger than the sum of the regions placed upon it. Then, the starting-point position of each region was assigned to a random base of the assigned chromosome, and then the stop position was calculated based on the size of that region, with checks in place to make sure the regions were non-overlapping and within the chromosome boundaries. Finally, the number of regions containing UMP genes was scored. This procedure was repeated 10000 times. The final distribution obtained in the test was shown as a histogram (Additional file 3: Figure S3).

The genome-wide significance threshold was determined using a randomisation test based on 1,000 permuted datasets as described by Churchill and Doerge [36]. Because of the computational demand of the randomisation testing, tests for epistasis were performed with $5 \mathrm{cM}$ spacing.

The proportion of the residual phenotypic variance that was explained by the detected QTL was calculated by the following equation:

$$
\operatorname{Var} \%=\left(M S_{R}^{\prime}-M S_{F}\right) / M S_{R}
$$

Here, $M S_{R}$ is the residual mean square of the reduced model (i.e. model (1) also including other QTL as cofactors or model (3), accordingly), $M S_{F}$ is the residual mean square of the full model (i.e. model (2) or (4), accordingly), and $M S_{R}$ is the residual mean square of the reduced model (only for model (1), it fit all covariates, but not any QTL for single-QTL analysis).

All of the statistical analyses were performed in the $\mathrm{R}$ statistical framework [31].

\section{Additional files}

Additional file 1: Table S1. Pearson correlations between all growthrelated traits used in the analysis. Table S2. Complete growth QTL results for all chromosomes in this study. Table S3. Complete epistatic QTL results for all detected significant pairs in this study. Table S4. Results of a two-dimensional scan including the suggestive interactions ( $F>8)$ detected in the scan. Table S5. Full list of UMP-related genes in the chicken genome. Table S6. Phenotypic averages for the body weight traits determined at different growth stages for the two divergent lines used as founders for the $F_{2}$ intercross.

Additional file 2: Figure S2. Two-locus genotype-phenotype maps for significant epistatic QTL pairs. Genotype-phenotype maps for the five significant two-locus interactions affecting: a) BW6, b) GR 4-8 (pair of regions in GGA6 and GGA25), c) GR 4-8 (pair of regions in GGA1 and GGA5), d) FCR 6-8, e) FCR 8-10. The letters $A$ and $B$ in the genotypes represent the line-of-origin of alleles from the founder lines HQLA and $H B$, respectively.

Additional file 3: Figure S3. Numerical distribution of the regions containing genes from the UMP pathway from a 10,000-time 
permutation test. The $x$-axis is the discrete number of regions (0-10) that contain genes belonging to the ubiquitin-mediated pathway. The $y$-axis shows the frequency of the corresponding region number observed in the test. The numbers above the rectangles are the actual counts of the corresponding observation from the10,000-time permutation test.

Additional file 4: Figure S4. Structure of the reciprocal cross between High Quality chicken Line A (HQLA) and Huiyang Beard chicken (HB) for QTL mapping. Using the last letter from the abbreviation of each line, we described the progeny between cocks from HQLA and hens from HB as "A $\times \mathrm{B}^{\prime}$ and vice versa. $\mathrm{F}_{0,}, \mathrm{~F}_{1}$ and $\mathrm{F}_{2}$ animals are labelled in orange, red and purple, respectively. Males are labelled with squares/rectangles, and females are labelled with circles/ovals. Descriptions of animals used in the cross are given in parentheses. The mating between F1 individuals are indicated with blue lines, and labelled with a serial number from 0-8 in dashed circles. In summary, we mated four HQLA cocks with $12 \mathrm{HB}$ hens, and four HB cocks with $12 \mathrm{HQLA}$ hens, yielding $399 \mathrm{~F}_{1}$ offspring. To balance the progeny of the eight $F_{0}$ cocks in the next generation, eight $F_{1}$ cocks (four $A \times B$ and four $B \times A$ ) and $48 F_{1}$ hens $(28 A \times B$ and $28 B \times$ A) were chosen for further crossing. Then, each cock from $A \times B$ was mated with six hens from $B \times A$ and vice versa.

Additional file 5: A txt file containing SNP chip data used in this study. Columns show the genotype data of each individual, while rows indicate the genotype data of the markers.

\section{Abbreviations}

AFW: Abdominal fat weight; BW: Live body weight; CAU_AB: Abbreviation for the China Agricultural University F2 intercross between line HQLA and line HB; CM: Centimorgan; CP: Crude protein; ER: ErbB signaling; FCR: Feed conversion ratio; GGA: Gallus gallus autosome; GR: Growth rate; HB: Huiyang Beard Chicken; HQLA: High Quality chicken Line A; ME: Metabolic energy; QTL: Quantitative trait locus/loci; SC: Shank circumference; SNP: Single nucleotide polymorphism; SL: Shank length; SW: Stomach weight: UMP: Ubiquitin mediated proteolysis.

\section{Competing interests}

The authors declare that they have no competing interests.

\section{Authors' contributions}

$\mathrm{NL}, \mathrm{ÖC}$, and XH conceived the project, designed and organized the study, $C L, H Q$, and DS established the experimental population and collected the data, ZS performaned the experiments and conducted the analysis, XS, ÖC and MEP assisted in the data analysis, ZS and MEP drafted the manuscript, ÖC edited the manuscript. All authors read and approved the final manuscript.

\section{Acknowledgements}

ZS would like to acknowledge support from the China Scholarship Council and also Dr Lucy Crooks for discussions and suggestions on data analysis. This work was supported by grants from the National High Technology Research and Development Program ("863" program) of China (Grant No. 2011AA100301), the joint Fund of the National Natural Science Foundation of China and the government of Guangdong Province (Grant No. U0831003), the National Natural Science Foundation of China (Grant No. 31272432), a EURYI Award to ÖC (DNR 2010-643), the Swedish Research Council (DNR: 521-2010-2652), FORMAS (DNR: 2010-643) and the Swedish Foundation for Strategic Research (DNR: F2006-0029:1).

\section{Author details \\ 'State Key Laboratory for Agro-Biotechnology, China Agricultural University, Beijing, People's Republic of China. ${ }^{2}$ Division of Computational Genetics, Department of Clinical Sciences, Swedish University of Agricultural Sciences (SLU), Uppsala, Sweden. ${ }^{3}$ State Key Laboratory of Livestock and Poultry Breeding, Guangzhou, People's Republic of China.}

Received: 24 September 2012 Accepted: 28 February 2013 Published: 6 March 2013

\section{References}

1. Ding HB, Xu RJ, Chen GA: The comparison of meat quality between Chinese indigenous chicken and broiler. Anim Husb Vet Med 2000, 32:16-18.
2. Tang $\mathrm{H}$, Gong $Y Z$, Wu CX, Jiang J, Wang Y, Li K: Variation of meat quality traits among five genotypes of chicken. Poult Sci 2009, 88(10):2212-8.

3. Zhao GP, Cui HX, Liu RR, Zheng MQ, Chen JL, Wen J: Comparison of breast muscle meat quality in 2 broiler breeds. Poult Sci 2011, 90(10):2355-9.

4. Jacobsson L, Park HB, Wahlberg P, Fredriksson R, Perez-Enciso M, Siegel PB, Andersson L: Many QTLs with minor additive effects are associated with a large difference in growth between two selection lines in chickens. Genet Res Camb 2005, 86(2):115-25.

5. Minvielle F, Kayang BB, Inoue-Murayama M, Miwa M, Vignal A, Gourichon D, Neau A, Monvoisin JL, Ito S: Microsatellite mapping of QTL affecting growth, feed consumption, egg production, tonic immobility and body temperature of Japanese quail. BMC Genomics 2005, 6:87.

6. Wahlberg P, Carlborg Ö, Foglio M, Tordoir X, Syvänen AC, Lathrop M, Gut $I G$, Siegel $P B$, Andersson $L$ : Genetic analysis of an $F_{2}$ intercross between two chicken lines divergently selected for body-weight. BMC Genomics 2009, 10:248.

7. Ankra-Badu GA, Shriner D, Bihan-Duval EL, Mignon-Grasteau S, Pitel F, Beaumont C, Duclos MJ, Simon J, Porter TE, Vignal A, Cogburn LA, Allison DB, Yi N, Aggrey SE: Mapping main, epistatic and sex-specific QTL for body composition in a chicken population divergently selected for low or high growth rate. BMC Genomics 2010, 11:107.

8. Matukumalli LK, Lawley CT, Schnabel RD, Taylor JF, Allan MF, Heaton MP, O'Connell J, Moore SS, Smith TP, Sonstegard TS, Van Tassell CP: Development and characterization of a high density SNP genotyping assay for cattle. PLoS One 2009, 4:e5350.

9. Ramos AM, Crooijmans RPMA, Affara NA, Amaral AJ, Archibald AL, Beever JE, Bendixen C, Churcher C, Clark R, Dehais P, Hansen MS, Hedegaard J, Hu Z-L, Kerstens HH, Law AS, Megens H-J, Milan D, Nonneman DJ, Rohrer GA, Rothschild MF, Smith TPL, Schnabel RD, Van Tassell CP, Taylor JF, Wiedmann RT, Schook LB, Groenen MAM: Design of a high density SNP genotyping assay in the pig using SNPs identified and characterized by next generation sequencing technology. PLoS One 2009, 4:e6524.

10. Groenen MA, Megens HJ, Zare Y, Warren WC, Hillier LW, Crooijmans RP, Vereijken A, Okimoto R, Muir WM, Cheng HH: The development and characterization of a 60K SNP chip for chicken. BMC Genomics 2011, 12(1):274.

11. Carlborg Ö, Haley CS: Epistasis: too often neglected in complex trait studies? Nat Rev Genet 2004, 5(8):618-25.

12. Steiner $\mathrm{CC}$, Weber JN, Hoekstra HE: Adaptive variation in beach mice produced by two interacting pigmentation genes. PLOS Biol 2008, 6(2):E36.

13. Noguera JL, Rodríguez $C$, Varona $L$, Tomàs $A$, Muñoz G, Ramírez $\mathrm{O}$, Barragán C, Arqué M, Bidanel JP, Amills M, Ovilo C, Sánchez A: A bi-dimensional genome scan for prolificacy traits in pigs shows the existence of multiple epistatic QTL. BMC Genomics 2009, 10:636.

14. Maccaferri M, Sanguineti MC, Corneti S, Ortega JLA, Salem MB, Bort J, DeAmbrogio E, Moral LFG, Demontis A, El-Ahmed A, Maalouf F, Machlab H, Martos V, Moragues M, Motawaj J, Nachit M, Nserallah N, Ouabbou H, Royo C, Slamaand A, Tuberosa R: Quantitative Trait Loci for Grain Yield and Adaptation of Durum Wheat (Triticum durum Desf.) Across a Wide Range of Water Availability. Genetics 2008, 178(1):489-511.

15. Malmberg RL, Held S, Mauricio AWR: Epistasis for Fitness-Related Quantitative Traits in Arabidopsis thaliana Grown in the Field and in the Greenhouse. Genetics 2005, 171(4):2013-2027.

16. Carlborg Ö, Kerje S, Schütz K, Jacobsson L, Jensenand P, Andersson L: A Global Search Reveals Epistatic Interaction Between QTL for Early Growth in the Chicken. Genome Res 2003, 13(3):413-421.

17. Carlborg Ö, Hocking PM, Burt DW, Haley CS: Simultaneous mapping of epistatic QTL in chickens reveals clusters of QTL pairs with similar genetic effects on growth. Genet Res 2004, 83(3):197-209.

18. Beavis WD, In Molecular Dissection of Complex Traits: QTL analyses: power, precision, and accuracy. New York: CRC press: 1st edition. Edited by Paterson AH; 1998:145-162.

19. Xu SZ: Theoretical basis of the Beavis effect. Genetics 2003, 165(4):2259-2268.

20. National Center for Biotechnology Information: [www.ncbi.nlm.nih.gov/].

21. KEGG: Kyoto Encyclopedia of Genes and Genomes.. www.genome.jp/kegg/.

22. Glickman MH, Ciechanover A: The Ubiquitin-Proteasome Proteolytic Pathway: Destruction for the Sake of Construction. Physiol Rev 2002, 82(2):373-428.

23. Marmor MD, Skaria KB, Yarden Y: Signal transduction and oncogenesis by ErbB/HER receptors. Int J Radiat Oncol Biol Phys 2004, 58(3):903-13.

24. Nitzan Z, Ben-Avraham G, Zoref Z, Nir I: Growth and development of the digestive organs and some enzymes in broiler chicks after hatching. $\mathrm{Br}$ Poult Sci 1991, 32:515-23. 
25. Lilja C, Marks HL: Changes in organ growth pattern associated with long-term selection for high growth rate in quail. Growth Dev Aging 1991, 55(4):219-24.

26. Chicken QTLdb:. http://www.animalgenome.org/cgi-bin/QTLdb/GG/index.

27. Xie L, Luo C, Zhang C, Zhang R, Tang J, Nie Q, Ma L, Hu X, Li N, Yang D, Zhang X: Genome-Wide Association Study Identified a Narrow Chromosome 1 Region Associated with Chicken Growth Traits. PLOS ONE 2012, 7(2):e30910.

28. Uemoto Y, Sato S, Odawara S, Nokata H, Oyamada Y, Taguchi Y, Yanai S, Sasaki O, Takahashi H, Nirasawa K, Kobayashi E: Genetic mapping of quantitative trait loci affecting growth and carcass traits in F2 intercross chickens. Poult Sci 2009, 88(3):477-82.

29. Liu X, Li H, Wang S, Hu X, Gao Y, Wang Q, Li N, Wang Y, Zhang H: Mapping Quantitative Trait Loci Affecting Body Weight and Abdominal Fat Weight on Chicken Chromosome One. Poult Sci 2007, 86(6):1084-89.

30. Rouzic AL, Álvarez-Castro JM, Carlborg Ö: Dissection of the Genetic Architecture of Body Weight in Chicken Reveals the Impact of Epistasis on Domestication Traits. Genetics 2008, 179(3):1591-9.

31. R Development Core Team: $R$ : A language and environment for statistical computing. Vienna, Austria: R Foundation for Statistical Computing; 2011. http://www.R-project.org. ISBN ISBN 3-900051-07-0, URL.

32. Green P, Fallsand $\mathrm{K}$, Crooks S: CRI-MAP Documentation version 2.4. Washington University School of Medicine St. Louis, MO: Cri-Map improved version is downloaded from; 1990. http://www.animalgenome.org/tools/share/crimap.

33. Groenen M, Wahlberg P, Foglio M, Cheng MH, Megens HJ, Crooijmans R, Besnier F, Lathrop M, Muir WM, Wong GKS, Gutand I, Andersson L: A high-density SNPbased linkage map of the chicken genome reveals sequence features correlated with recombination rate. Genome Res 2009, 19(3):510-519.

34. Haley CS, Knott SA, Elsen JM: Mapping Quantitative Trait Loci in Crosses between Outbred Lines Using Least Squares. Genetics 1994, 136(3):1195-1207.

35. Crooks L, Nettelblad C, Carlborg O: An Improved Method for Estimating Chromosomal Line Origin in QTL Analysis of Crosses Between Outbred Lines. G3 2011, 1(1):57-64.

36. Churchill GA, Doerge RW: Empirical Threshold Values for Quantitative Trait Mapping. Genetics 1994, 138(3):963-971.

doi:10.1186/1471-2164-14-151

Cite this article as: Sheng et al:: Genetic dissection of growth traits in a Chinese indigenous $\times$ commercial broiler chicken cross. BMC Genomics 2013 14:151.

\section{Submit your next manuscript to BioMed Central and take full advantage of:}

- Convenient online submission

- Thorough peer review

- No space constraints or color figure charges

- Immediate publication on acceptance

- Inclusion in PubMed, CAS, Scopus and Google Scholar

- Research which is freely available for redistribution 